\title{
A Qualitative Acceleration Model Based on Intervals
}

\author{
Ester Martinez-Martin ${ }^{\mathrm{a}}$, Maria Teresa Escrig ${ }^{\mathrm{b}}$, Angel P. del Pobil ${ }^{\mathrm{a}}$ \\ ${ }^{a}$ Robotic Intelligence Lab, Dept. Engineering and Computer Science, Universitat Jaume I, Castellón, Spain \\ ${ }^{\mathrm{b}}$ Cognitive Robot, S.L. Parque Científico, Tecnológico y Empresarial, Universitat Jaume I, Castellón, Spain
}

KEYWORD

Model-based reasoning

Qualitative reasoning

Spatial reasoning

\section{Introduction}

Recent research is interested in building autonomous systems helping people in their daily tasks, especially when they are tedious and/or repetitive. These common tasks can involve poorly defined situations. On this matter, humans have a remarkable capability to solve them without any measurements and/or any computations. Familiar examples are parking a car, cooking a meal, or summarizing a story. That is, people make decisions mostly based on perceptual information, rather than accurate measurements [ZADEH, 2001]. Thus, qualitative reasoning properly fits this problem since it works on representation formalisms close to human conceptual schemata for reasoning about the surrounding physical environment (e.g. [WESTPHAL \& WÖLFL, 2009]).

Focusing on safety in Robotics, a key point is detecting and following all-embracing elements in order to avoid collisions, especially when they are human beings. Although some devices have been developed to avoid collisions, they considerably restrict the system's autonomy and flexibility. In addition, they present imprecise data and, for that reason, a qualitative model is required. In this paper, we present a new qualitative model of acceleration combined with orientation, which provides surrounding element's pose, allowing the system to properly avoid potential collisions. In qualitative spatial reasoning, a particular aspect of the physical world, a magnitude, is considered. That is, a system of qualitative relationships between entities that cover that aspect of the world to some degree is developed. Examples of that can be found in many disciplines (e.g. geography [VAN DE WEGHE et al., 2006], psychology [KNAUFF et al., 2004], ecology [CIOACA et al., 2009], biology [KING et al., 2005], robotics [LIU et al., 2008] and Artificial Intelligence [COHN \& HAZARIKA, 2001]). Actually, a qualitative representation of a magnitude results from an abstraction process and it has been defined as that representation that makes only as many distinctions as necessary to identify objects, events, situations, etc. in a given context for that magnitude in [RENZ \& NEBEL, 2007]. The way to define those distinctions depends on two different aspects:

1. the level of granularity. In this context, granularity refers to a matter of precision in the sense of the amount of information which is included in the representation

2. the distinction between comparing and naming magnitudes (as stated in [CLEMENTINI et al., 1997]). This distinction refers to the usual comparison between absolute and relative. From a spatial point of view, this controversy corresponds to the way of representing the relationships 
among objects (see Fig. 1). From the distinction between absolute and relative pointed out by [LEVINSON, 2003], an object $\boldsymbol{b}$ is any compared relationship to another object $\boldsymbol{a}$ from the same Point of View $(\boldsymbol{P} \boldsymbol{V})$ when comparing magnitudes are considered. It is worth noting that the comparison depends on the orientation of both objects with respect to (wrt) the $\boldsymbol{P V}$, since objects $\boldsymbol{a}$ and $\boldsymbol{b}$ can be at any orientation wrt the $\boldsymbol{P} \boldsymbol{V}$. On the other hand, naming magnitudes divides the magnitude of any concept into intervals (sharply or overlapped separated, depending on the context) such that qualitative labels are assigned to each interval. Note that the result of reasoning with this kind of regions can provide imprecision. This imprecision will be solved by providing disjunction in the result. That is, if an object can be found in several qualitative regions, $\boldsymbol{q}_{i}$ or $\boldsymbol{q}_{i+1}$ or $\ldots$ or $\boldsymbol{q}_{\boldsymbol{n}}$, then all possibilities are listed as follows $\left\{\boldsymbol{q}_{i}, \boldsymbol{q}_{i+1}, \ldots, \boldsymbol{q}_{n}\right\}$ by indicating this situation

From that starting point, and on the way to develop intelligent abilities to solve some service robotics problems, in this paper, we present a qualitative naming acceleration model including its qualitative representation, the reasoning process and a real robotic application. With that aim, the structure of this paper is as follows: the proposed qualitative acceleration model is analysed in Section 2, while a practical application is described in Section 3. Finally, some conclusions and future work are presented in Section 4.

\section{Qualitative Acceleration Model}

The acceleration is the physical concept that measures how an object's speed or direction changes over time. Physically, it can be defined as:

$$
\text { Acceleration }=\frac{\text { Velocity }}{\text { Time }}=\frac{\text { Space }}{\text { Time }^{2}}
$$

\subsection{Representation}

The first issue to be solved concerns how acceleration is represented. So, from the previous acceleration definition and focusing on developing its qualitative naming model, the acceleration representation will consist of three elements:

1. number of objects implied in each relation (i.e. arity). From the physical definition of acceleration, the relationships to be defined imply two objects such that an object acts as reference and the other one is referred

2. the set of acceleration relations between objects. It depends on the considered level of granularity. In a formal way, this set of relations is expressed by means of the definition of a Reference System (RS) composed of:

- a set of qualitative symbols in increasing order represented by $\mathrm{Q}=\left\{\mathrm{q}_{0}, \mathrm{q}_{1}, \ldots, \mathrm{q}_{\mathrm{n}}\right\}$, where $\mathrm{q}_{0}$ is the qualitative symbol closest to the Reference Object $(\mathrm{RO})$ and $\mathrm{q}_{\mathrm{n}}$ is the one furthest away, going to infinity. Here, by cognitive considerations, the acceptance
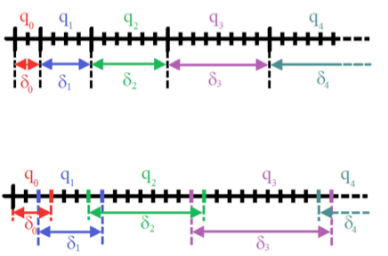

NAMING MAGNITUDE

Fig. 1. An example of compared distances as represented in [Escrig \& Toledo, 2001] (left) and an example of structure relations in naming magnitudes with sharply and overlapped separated qualitative areas (right) 
areas have been chosen in increasing size

- $\quad$ the structure relations, $\Delta_{\mathrm{r}}=\left\{\delta_{0}, \delta_{1}\right.$, $\left.\ldots, \delta_{n}\right\}$, describe the acceptance areas for each qualitative symbol $\mathrm{q}_{\mathrm{i}}$. So, $\delta_{0}$ corresponds to the acceptance area of qualitative symbol $q_{0} ; \delta_{1}$ to the acceptance area of symbol $\mathrm{q}_{1}$ and so on. These acceptance areas are quantitatively defined by means of a set of close or open intervals delimited by two extreme points: the initial point of the interval $j, \delta_{\mathrm{j}}^{\mathrm{i}}$, and the ending point of the interval $j, \delta_{\mathrm{j}}^{\mathrm{e}}$, such that the structure relations are rewritten as:

$\left\{\Delta r=\left\{\left[\delta_{0}^{i}, \delta_{0}^{e}\left[,\left[\delta_{1}^{i}, \delta_{1}^{e}\left[, \ldots,\left[\delta_{n}^{i}, \delta_{n}^{e}[\}\right.\right.\right.\right.\right.\right.\right.$ if open intervals are considered

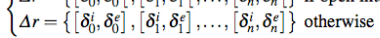

Therefore, the acceptance area of a particular acceleration entity, $\operatorname{AcAr}$ (entity), is $\delta_{\mathrm{j}}$ if its value is between the initial and ending points of $\delta_{j}$, that is,

$$
\delta_{j}{ }^{i} \leq \text { value (entity) } \leq \delta_{j}{ }^{\mathrm{e}}
$$

- the operations. The number of operations associated to a representation corresponds to the possible change in the PV. In this case, as only two objects are implied in the acceleration relationships, only one operation can be defined: inverse

Note that a particularity of acceleration is that the values for the intervals in which the workspace is divided into, can be both positives and negatives and this feature has to be considered when the reasoning process is designed.

\subsection{The Basic Step of the Inference Process}

The Basic Step of the Inference Process (BSIP) for the acceleration concept can be defined as: given two acceleration relationships between three spatio-temporal entities $\boldsymbol{a}, \boldsymbol{b}$ and $\boldsymbol{c}$, we want to find the acceleration relationship between the two entities that is not initially given. However, it is important to take into account that the relative movement of the implied objects can be at any direction. For that reason, the BSIP is studied integrating the acceleration concept with a qualitative orientation model. Note that, for this case, the qualitative orientational model of Freksa [FREKSA, 1992] has been redefined as depicted in Fig. 2 since the RO is always on the object $\boldsymbol{b}$. In that way, it is possible to reason with the extreme angles of the defined structure relations for the Orientation Reference System (ORS).

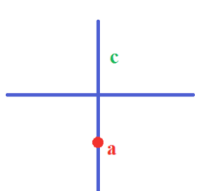

$$
\begin{aligned}
\mathbf{O R S}= & \left\{\mathbf{Q}_{\circ}, \Delta \mathbf{r}_{\circ}\right\} \\
\mathbf{Q}_{0}= & \{\text { front-left (fl), straight-front (sf), front-right (fr), } \\
& \text { left (l), none (n), right (r), } \\
& \text { back-left (bl), straight-back (sb), back-right (br) }\} \\
\Delta \mathbf{r}_{0}= & \{190,180[,[90,90],] 0,90[, \\
& {[180,180],,[0,0], }
\end{aligned}
$$$$
\text { ]180, 270[, [270, 270], ]270, 360[\} }
$$

Fig. 2. Redefinition of the Orientation Reference System (ORS) of [Freksa, 1992] by means of its set of qualitative symbols $\left(\mathrm{Q}_{0}\right)$ and its structure relations $\left(\Delta_{0}\right)$

Given that qualitative areas are defined by intervals, we use the two operations to add and subtract qualitative intervals presented in our previous work [MARTÍNEZ-MARTín et al., 2012]. In particular, the functions to be performed are qualitative_sum (obtains the sum of two qualitative intervals), qualitative_difference (provides the subtraction of two qualitative intervals), Find_UB_qualitative_sum \} (obtains the qualitative interval corresponding to the Upper Bound (UB) of the qualitative sum of two qualitative intervals) and Find_LB_qualitative_difference (provides the qualitative interval corresponding to the Lower Bound (LB) of the qualitative subtraction of two qualitative intervals). In addition, five new functions are defined: pythagorean_theorem_LB and pythagorean_theorem_UB that obtain the qualitative interval respectively corresponding to the lower and upper bounds when the Pythagorean theorem is applied; intermediate_orientation provides the orientations existing between the two ones given as input (e.g. intermediate_orientation(right, straight-front) will be front-right); open_interval, from an orientation defined with a closed interval and another with an open interval, returns that corresponding to an open interval; and, all_orientation_relationships returns all the defined qualitative orientations. 


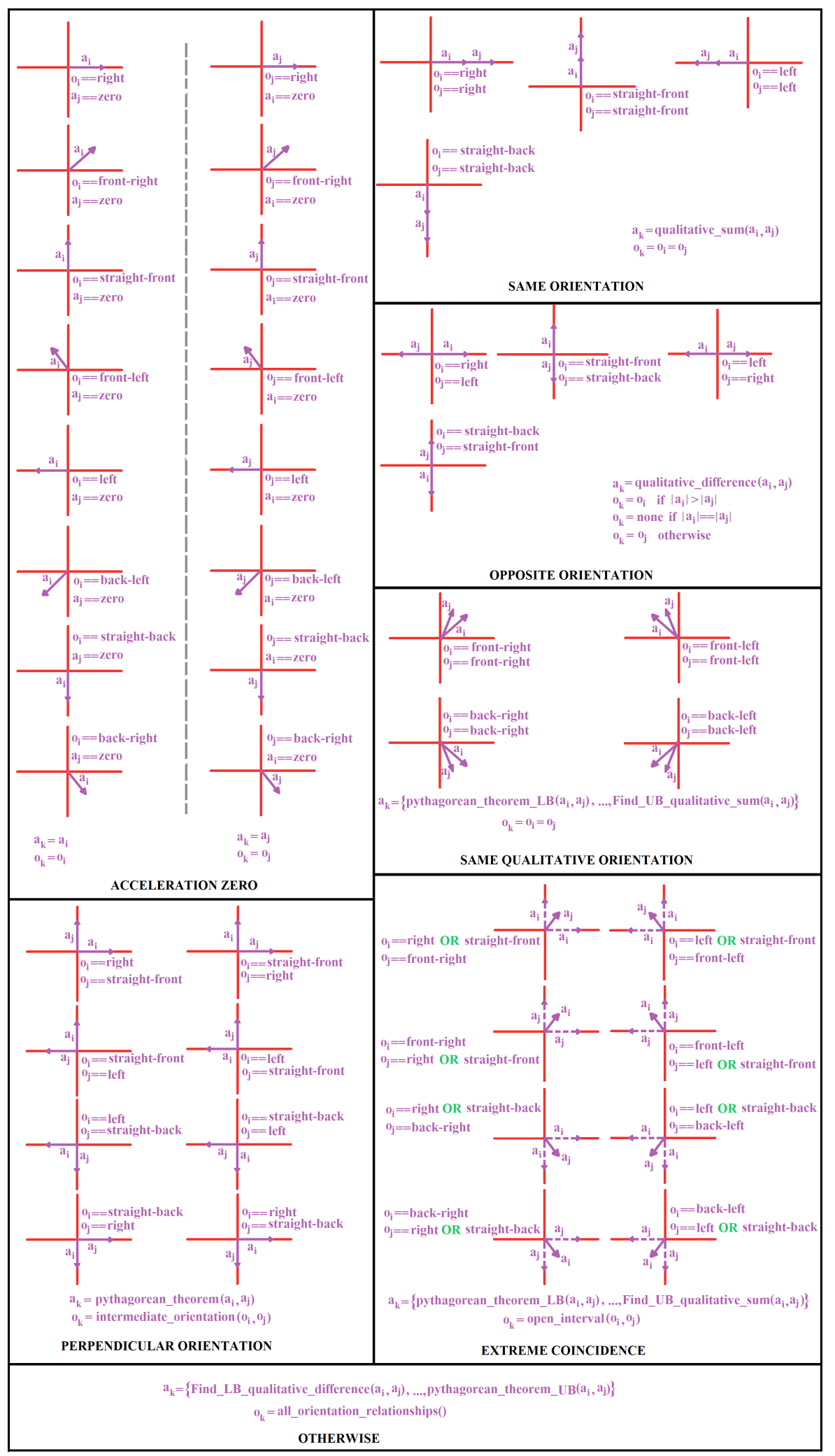

Fig. 3. Graphical resolution of the BSIP for acceleration, where $a_{i}$ and $a_{j}$ represents the acceleration relationships given as input with their corresponding orientation relationships $\left(\mathrm{o}_{i}\right.$ and $\left.o_{j}\right)$; and $a_{k}$ and $o_{k}$ are the resulting acceleration and orientation relationships 
Therefore, the BSIP for acceleration has been solved as follows (see Fig. 3): when any acceleration relationship is zero, both acceleration and orientation will be equal to the other involved relationship. When the two acceleration relationships have the same orientation, the resulting relationship has the same orientation and its value corresponds to the qualitative sum of both relationships. On the contrary, if the relationships have an opposite orientation, the resulting relationship will be obtained as their qualitative difference and its orientation will be equal to that of the highest acceleration value (in absolute values). On the other hand, in the case both relationships have the same orientation but it corresponds to an open interval, the resulting relationship has the same orientation, although its value will be a disjunction of acceleration relationships from the result of applying the Pythagorean theorem to the UB qualitative sum. When the orientation relationships corresponds to an open and a close interval such that one extreme of an interval matches up with an extreme of the other interval, then the resulting relationship will have the orientation of the open interval, while its value will be obtained from the Pythagorean theorem and the qualitative sum. The last special case refers to the case two orientation relationships are perpendicular. In that situation, the resulting relationship results from the Pythagorean theorem, whereas its orientation is the orientation relationship between the orientations of the initial relationships. Finally, the remaining situations are solved by means of qualitative difference and the Pythagorean theorem. With regard to its orientation, it corresponds to all the possible orientation relationships.

\subsection{The Complete Step of the Inference Process}

From the BSIP definition, the Complete Inference Process $(\boldsymbol{C I P})$ can be defined. So, mainly, it consists of repeating the BSIP as many times as possible with the initial information and the information provided by some BSIP until no more information can be inferred. As knowledge about relationships between entities is often given in the form of constraints, the CIP can be formalized as a
Constraint Satisfaction Problem (CSP). So, the computation of the full inference process for qualitative acceleration can be viewed as an instance of the CSP. So, on way of solving this acceleration inference process, a Constraint Logic Programming (CLP) program extended with Constraint Handling Rules (CHRs) has been developed.

\section{A Practical Application}

A real application of the proposed method is presented. In this case, the qualitative acceleration model has been implemented on a mobile robot. The aim of this system is to assist human beings in performing a variety of tasks such as carrying person's tools or delivering parts. One of the major requirements of such robotic assistants is the ability to track and follow a moving person through a nonpredetermined, unstructured environment. To achieve this goal, two different tasks have to be performed: person recognition and segmentation from the surrounding environment, and motion control to follow the person using the recognition results. In particular, we have focused on developing the qualitative reasoning method to achieve the second task. For that, an indoor pan-tilt-zoom (PTZ) camera was mounted on a Pioneer 3-DX mobile platform without restricting its autonomy and flexibility. The core of the PTZ system is a Canon VC-C4 analog colour camera with a resolution of $320 \times 240$ pixels, which is integrated with the mobile platform hardware. So, on the one hand, the system knows both its acceleration and its orientation through the information gathered from its motors. On the other hand, an image processing based on optical flow provides an estimation of the acceleration and orientation relationships corresponding to the person to be followed. Therefore, from these two relationships (the one obtained by the robotic system itself and the other corresponding to the person from image processing), the system is able to determine the required accelerationorientation relationship that allows it to know the required trajectory change to properly follow and assist that person. An example of the obtained results can be seen in Fig. 4 . 


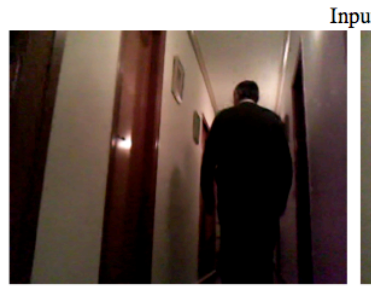

Qualitative classification Acceleration Relationships
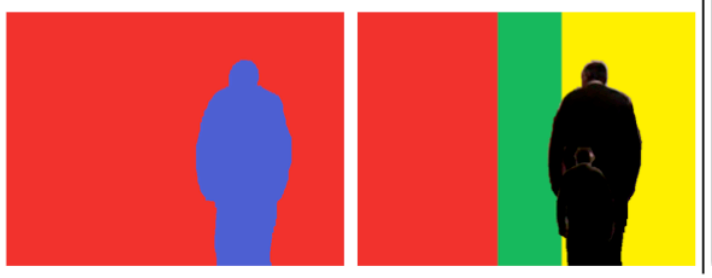

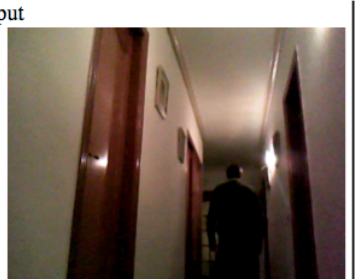

Orientation Relationships

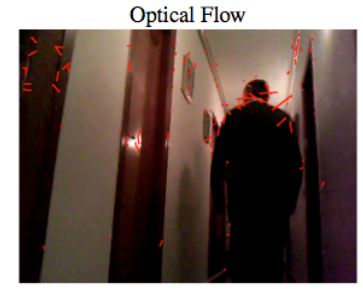

Snapshot program running

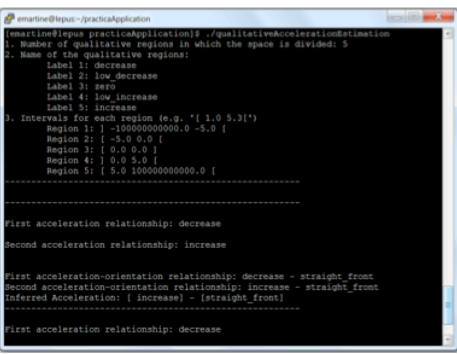

Fig. 4. Results obtained with the real robot when the qualitative acceleration relationships are labelled as $\mathrm{Q}=\{$ decrease, low_decrease, zero, low_increase, increase $\}$ and the orientation relationships correspond to the modified Freksa's approach previously defined, such that $f l$ is coded by red, $s f$ by green and $f r$ by yellow

\section{Conclusions and Future Work}

In this paper, we have developed a qualitative model for physical acceleration such that acceleration and orientation are combined, the basic step of the inference process is expressed in terms of qualitative sums and differences, and, given that knowledge about relationships between entities is often provided in the form of constraints, the complete inference process is formalized as a Constraint Satisfaction Problem $(C S P)$.

The qualitative acceleration model defined in these terms allows to automatically estimating people's pose around the system and, therefore, avoiding collisions.
This results in safer and more accurate robotic systems, in spite of sensor imprecision.

As future work we will investigate the development of new qualitative models based on intervals of aspects such as: time, weight, body sensations, etc. to provide robots with intelligent abilities to solve service robotics problems.

\section{Acknowledgments}

This research was partly supported by Ministerio de Ciencia e Innovación (DPI201127846), by Generalitat Valenciana (PROMETEO/2009/052) and by Fundació Caixa Castelló-Bancaixa (P1-1B2011-54). 


\section{$6 \quad$ References}

[CIOACA et al., 2009]

[CLEMENTINI et al., 1997]

[COHN \& HAZARIKA, 2001]

[ESCRIG \& TOLEDO, 2001]

[FREKSA, 1992]

[KING et al., 2005]

[KNAUFF et al., 2004]

[LEVINSON, 2003]

[LIU et al., 2008]

[MARTÍNEZ-MARTÍN et al., 2012]

[RENZ \& NEBEL, 2007]

[VAN DE WEGHE et al., 2006]

[WESTPHAL \& WÖLFL, 2009]

[ZADEH, 2001]
Cionca, E., Linnebank, F., Bredeweg, B., \& Salles, P.: A qualitative reasoning model of algal bloom in the danube delta biosphere reserve (ddbr). Ecol Informatics, 4(5-6): 282-298, 2009

Clementini, E., Felice, P.D., HernándeZ, D.: Qualitative representation of positional information. AI, 95(2): 317-356, 1997 COHN, A., \& HAZARIKA, S.: Qualitative spatial representation and reasoning: An overview. Fundamenta Informaticae, 46(1-2): 1-29, 2001

ESCRIG, M., \& TOLEDO, F. Reasoning with compared distances at different levels of granularity. In: CAEPIA. Gijón, Spain, 2001

FREKSA, C. Using orientation information for qualitative spatial reasoning. In: Theories and Methods of Spatio-Temporal Reasoning in Geographic Space, LNCS, vol. 639. Springer-Verlag, Berlin, Germany, 1992

King, R., Garrett, S., \& Coghill, G. On the use of qualitative reasoning to simulate and identify metabolic pathways. Bioinformatics, 21(9): 2017-2026, 2005

Knauff, M., Strube, G., Jola, C., Rauh, R., \& Schlieder, C.: The psychological validity of qualitative spatial reasoning in one dimension. Spatial Cognition \& Computation, 4(2): 167-188, 2004

LEVINSON, S. Space in Language and Cognition. Explorations in Cognitive Diversity. Cambridge University Press, UK, 2003

LiU, H., Brown, D., \& CoghiLl, G.: Fuzzy qualitative robot kinematics. IEEE Trans. on Fuzzy Systems, 16(3): 808-822, 2008 Martínez-Martín, E., Escrig, M.T., \& DElPobil, A. A General Framework for Naming Qualitative Models Based on Intervals, AISC, vol. 151, pp. 681-688. Springer-Verlag, 2012

RENZ, J., \& NEBEL, B. Qualitative Spatial Reasoning Using Constraint Calculi, pp. 161-215. Springer Verlag, Berlin, Germany, 2007

VAn de Weghe, N., Cohn, A., De Tré, G., \& De Maeyer, P.: A qualitative trajectory calculus as a basis for representing moving objects in geographical information systems. Control and Cybernetics, 35(1): 97-119, 2006

WestPhal, M., \& WöLfL, S. Qualitative csp, finite csp, and sat: Comparing methods for qualitative constraint-based reasoning. In: IJCAI, pp. 628-633, 2009

ZADEH, L. A new direction in AI. Toward a computational theory of perceptions. AI Magazine, 22(1): 73-84, 2001 